\title{
Deteriorating rural ponds : A threat to overseas migratory wetland birds in Kurukshetra suburbs, Haryana, India
}

\section{Tirshem Kumar Kaushik* and Rohtash Chand Gupta}

Department of Zoology, Kurukshetra University, Kurukshetra -136119 (Haryana), INDIA

*Corresponding author. E-Mail:-tarshemkaushik@ rediffmail.com

Received: May 04, 2014; Revised received: August 03, 2014; Accepted: October 29, 2014

\begin{abstract}
The present study addresses the problem of ongoing destruction of traditional age old rural wetlands in Haryana in the last 10 years. It is a grave issue which stands accelerated due to anthropogenic unawareness and in deliberate destruction. In all, 59 species of wetland birds belonging to 10 orders and 17 families were recorded from Palwal rural pond in Kurukshetra district in India during 2005-2012. The presence and that too in higher numbers of Purple Moorhen Porphyrio porphyrio and Common Moorhen Gallinula chloropus hints towards the over excessive growth of hyacinth and water reeds thus making it an ideal place for these birds which are the harbingers of total eutrophication of a certain water body. The other birds seen in the pond are White-breasted Waterhen Amaurornis phoenicurus, Red-wattled Lapwing Vanellus indicus, Large Egret Ardea alba and Median Egret Mesophoyx intermedia. Pond Herons Ardeola grayii were in few numbers. Black winged Stilts Himantopus himantopus were in more number thus indicating the presence of sludge in water and its shallowness. Cattle egrets were in large number indicating its negative character. The lackluster attitude of Govt. of Haryana will result in its total destruction in 5-10 years. Similar situation may be extended to nearby village ponds to worsen their condition too. Ramsar Convention (1971) pleads for preservation, protection and conservation of wetlands of all hues. Village ponds in Haryana should be revived by seeking co-operation from the Head Quarter of Ramsar Convention at Switzerland.
\end{abstract}

Keywords: Kurukshetra, Village pond, Winter migratory birds

\section{INTRODUCTION}

Haryana state has approximately 6759 villages, considering that every village has 4-5 ponds, there will be approximately 25000 ponds. These rural ponds are the natural water-sheds in rural India since time immemorial. Actually, entire rainy water of the village flows down to these ponds in a natural way. These ponds serve many purposes of the villagers in day to day use and also for some very important utility purposes in specific seasons. The traditional wells are located nearby to these ponds which would recharge the ground water used incessantly for domestic purpose as well as for irrigating agriculture fields. The regulation of this very utility in a typical village was self driven and flawless, capable of serving the needs throughout the year. Most interestingly, these ponds are very convenient spots for sojourning wetland winter visiting birds in and around Kurukshetra (Gupta and Kaushik, 2010b). However, a very serious negative process has driven into this self driven natural prevalence of water in ponds in Kurukshetra especially after 2005. It is a recent phenomenon having alarming consequences on the winter visitor birds. These ponds are over burdened by the ongoing fast process of eutrophication. This causes a direct threat to winter visiting migratory birds. In this paper a case study of Palwal village pond has been followed for 7 years, which has highlighted a major issue of threats to ponds and the associated birds. Extensive work has been done on these lines in the Department of Zoology, Kurukshetra University, Kurukshetra (Gupta et al. 2009, 2010 a-c; Gupta and Kaushik, 2010a-e; Gupta and Kaushik, 2011a-b; Gupta et al. 2012; Gupta and Kaushik, 2012; Gupta and Kaushik, 2013a-b; Kaushik and Gupta, 2013; Kaushik and Gupta, 2014).

The situation of all wetlands throughout of the world is enigmatic. Against the backdrop of the sermons of Ramsar Convention, several paradoxes surround the existence of wetlands has also the practical applicability of policies, measures and mandated wetland revival projects. Today the sum total of conditions has declared an onslaught on the very existence of the centuries old useful ponds in villages in Haryana. This may be attributed to fish farming, land filling ponds in a clandestine manner followed by encroachment for house building (Gupta et al.2012, Gupta and Kaushik, 2012). On top of these, is the abetting of eutrophication process linked with invasion of ponds by massive hyacinth plants that grow virtually everywhere in the ponds and partly by dumping of organic wastes in the vicinity of the ponds, principally the cow dung in huge mounds which turn the water into poisonous volume 
for phytoplanktons, zooplanktons, nektons thus completely demolishing the trophical pyramids. The present study explains a widespread destruction of wetlands and wetland associated diversity of winter migratory birds which migrate to Haryana and other parts of northern India.

\section{MATERIALS AND METHODS}

Study area: The locus of present study is a vast village pond nearby Palwal Village in Tehsil Thaneasr district Kurukshetra, India (Latitude $29^{0} 52^{\prime}$ to $30^{0} 12^{\prime}$ and Longitude $76^{0} 26^{\prime}$ to $77^{\circ} 04^{\prime}$ ). Palwal village is located on a high platform compared to the level of the village pond and hence is an ideal depression to receive huge rainy waters of the village. As a result, it was healthier pond upto 2000-2005 spanning on over 20-25 acres of land. Its vast expanse still imparts it a shape similar to a lake. On its southern side lies the village boundary and its other peripheral outskirts are bounded by the agriculture fields. It is ancient, perennial pond recurring rainy run-off water from the nearby village. The mid depth accounts 8-10 feet. Its catchment area is 400-500 sq. feet. and the entire water sheet supports lush green hyacinth. It used to be a healthy perennial pond rich in diversity of birds, tortoises, molluscs and aquatic plants like Chara, Potomogeton and Lotus etc. However, it is important to note that, the pond is a ready source of recharging the ground water. But presently, this pond is in a very bad shape. The periphery of the pond is completely eutrophied. It shallow water regions ridden with over excessive growth of reeds and reed plants.

Methodology: The Palwal village pond was under surveillance since 2005 onwards in a continuous manner. Prior to this, this pond was glanced character in terms of shrinking water sheet, over burdened by hyacinth and water reeds in 2008. Actually, between 2005-2008 regular visits were made to comprehend the spectrum of winter visiting birds. However since 2008 the situation catapulted this healthy pond into a eutrophied water sheet. In these conditions it is being regularly visited. Extensive photography has been done with Nikon Coolpix P500 Camera, to record the migratory as well as the resident birds. Birds were identified with the aid of reference books viz. Ali and Ripley (1987), Ali (1996), Grimmet et al. (1998) and Kumar et al. (2005). The nomenclature follows Manakadan and Pittie (2001).

\section{RESULTS AND DISCUSSION}

It is evident from Table 1 that the diversity of wetland birds in Palwal pond in Kurukshetra district was very rich representing 10 orders 17 families and their collective number was 59 . Out of 59 species of birds, 30 species were winter migratory, 17 species resident, 10 species were local migratory and two species of birds were summer migratory. Gupta and Kaushik (2013b) reported 36 species of wetland birds belonging to 8 orders and 11 families from Brahmsarovar wetland in Kurukshetra district in Haryana. Also, Kaushik and Gupta (2013) recorded 60 species of wetland birds from Asan barrage in Uttarakhand state in northern Haryana. At the same time, Gupta and Kaushik (2013a) observed 58 species of birds belonging to 10 orders and 18 families from Nigdu village pond in Karnal district in Haryana. It is pertinent to mention that Pintail Snipe is only spotted from Palwal village pond in Kurukshetra district in Haryana. Compared to this, the situation between 2009 -2012 seems to have been obliterated completely as collective numbers have collapsed to merely 36 depicting the total failure of the pond to attract wetland birds (Table 1).

The present studies hint towards the fact that Palwal village pond is receding towards disastrous eutrophication (Figs. 1-3, Plate 1). Photographic evidence in plate 1 proves that hyacinth has overtaken this, once upon a time a very healthy pond completely in all direction and across the expanse foot-by-foot (Figs. 1-3, Plate 1). Eutrophied land of the pond stands encroached upon by the land mafia where house construction is going on with favour in a clandestine way (Figs. 10-11, Plate 1). Gupta and Kaushik (2012) reported that the siltation of ponds with adjoining areas, soil coming in with rainwater is a very serious cause of stratification of rural ponds in Haryana.

About 2-4 Square kilometer area thus eutrophied has already been encroached upon by land mafia and new housing colony has already been initiated (Figs. 10-11, Plate 1). Considering the pace of construction of dwelling houses, the entire pond will be a new colony of houses. The only relic and remnant of this age old pond will be the Temple in the south direction (Plate1). Until 2008, there was a very diversity of resident wetland birds, including, Pond Herons Ardeola grayii, huge numbers of Black winged Stilts Himantopus himantopus, Common Moorhens Gallinula chloropus, Bronze-winged Jacana Metopidius indicus, Red-wattled Lapwing Vanellus indicus, Yellow wattled Lapwing Vanellus malabaricus, Grey Herons Ardea cinerea, large Egrets Ardea alba and Median Egrets Mesophoyx intermedia (Table-1). Gupta and Kaushik (2013) reported birds like Painted Stork Mycteria leucocephala, White-necked Stork Ciconia episcopus, Black-necked Stork Ephippiorhynchus asiaticus, Spotted Greenshank Tringa guttifer, Eurasian Spoonbill Platela leucorodia, Bar-headed Goose Anser indicus and Greylag Goose Anser anser from Nighdhu village pond in Karnal but these birds could not be observed from Palwal village ponds in Kurukshetra district in Haryana.

Most importantly, Palwal Pond used to be a very significant wintering harbor for huge flocks of migratory birds like Mallard Anas platyrhynchos, Northern Pintail Anas acuta, Northern Shoveller Anas clypeata, Common Pochard Aythya ferina, Gadwall 
Table 1. Wetland birds diversity of Palwal village pond in Kurukshetra district in Haryana, India.

\begin{tabular}{|c|c|c|c|c|}
\hline S.N. & Common Name & Scientific Name & $\begin{array}{l}\text { Residential } \\
\text { status }\end{array}$ & Remarks \\
\hline & Podicipediformes & & Podicipedidae & \\
\hline \multirow[t]{2}{*}{1} & Little Grebe & $\begin{array}{l}\text { Tachybaptaus rufficollis } \\
\text { (Pallas, 1764) }\end{array}$ & $\mathrm{R}$ & $\begin{array}{l}\text { Recorded throughout the study } \\
\text { period (2005-2012) }\end{array}$ \\
\hline & Pelecaniformes & & Phalacrocorac & lae \\
\hline 2 & Little Cormorant & $\begin{array}{l}\text { Phalacrocorax niger } \\
\text { (Vieillot, 1817) }\end{array}$ & $\mathrm{R}$ & Recorded during 2005-2008 \\
\hline 3 & Indian Shag & $\begin{array}{l}\text { Phalacrocorax fuscicollis } \\
\text { Stephens, } 1826\end{array}$ & LM & Recorded during 2005-2008 \\
\hline \multirow[t]{2}{*}{4} & Great Cormorant & $\begin{array}{l}\text { Phalacrocorax carbo } \\
\text { (Linnaeus, 1758) }\end{array}$ & LM & Recorded during 2005-2008 \\
\hline & Ciconiiformes & & Ardeidae & \\
\hline 5 & Little Egret & $\begin{array}{l}\text { Egretta garzetta } \\
\text { (Linnaeus, 1766) }\end{array}$ & WM & Recorded during 2005-2008 \\
\hline 6 & Grey Heron & $\begin{array}{l}\text { Ardea cinerea } \\
\text { Linnaeus, } 1758\end{array}$ & WM & Recorded during 2005-2008 \\
\hline 7 & Purple Heron & $\begin{array}{l}\text { Ardea purpurea } \\
\text { Linnaeus, } 1766\end{array}$ & WM & Recorded during 2005-2008 \\
\hline 8 & Large Egret & $\begin{array}{l}\text { Casmerodius albus } \\
\text { (Linnaeus 1758) }\end{array}$ & LM & Recorded during 2005-2008 \\
\hline 9 & Median Egret & $\begin{array}{l}\text { Mesophoyx intermedia } \\
\text { (Wagler 1829) }\end{array}$ & LM & $\begin{array}{l}\text { Recorded throughout the study } \\
\text { period (2005-2012) }\end{array}$ \\
\hline 10 & Cattle Egret & $\begin{array}{l}\text { Bubulcus ibis } \\
\text { (Linnaeus, 1758) }\end{array}$ & $\mathrm{R}$ & $\begin{array}{l}\text { Recorded throughout the study } \\
\text { period }(2005-2012)\end{array}$ \\
\hline \multirow[t]{2}{*}{11} & Indian Pond-Heron & $\begin{array}{l}\text { Ardeola grayii } \\
\text { (Sykes, 1832) }\end{array}$ & $\mathrm{R}$ & $\begin{array}{l}\text { Recorded throughout the study } \\
\text { period (2005-2012) }\end{array}$ \\
\hline & & & Ciconiidae & \\
\hline \multirow[t]{2}{*}{12} & Asian Openbill-Stork & $\begin{array}{l}\text { Anastomus oscitans } \\
\text { (Boddaert, 1783) }\end{array}$ & LM & Recorded during 2005-2008 \\
\hline & & & \multirow[t]{2}{*}{ Threskior } & nithidae \\
\hline 13 & Black Ibis & $\begin{array}{l}\text { Pseudibis papillosa } \\
\text { (Temminck, 1824) }\end{array}$ & & Recorded during 2009-2012 \\
\hline & Anseriformes & \multicolumn{3}{|c|}{ Anatidae } \\
\hline 14 & $\begin{array}{l}\text { Lesser Whistling } \\
\text { duck }\end{array}$ & $\begin{array}{l}\text { Dendrocygna javanica } \\
\text { (Horsfield, 1821) }\end{array}$ & SM & $\begin{array}{l}\text { Recorded throughout the study } \\
\text { period }(2005-2012)\end{array}$ \\
\hline 15 & Rudy Shelduck & $\begin{array}{l}\text { Tadorna ferruginea } \\
\text { (Pallas 1764) }\end{array}$ & WM & Recorded during 2011-2012 \\
\hline 16 & Comb Duck & $\begin{array}{l}\text { Sarkidiornis melanotos } \\
\text { (Pennant, 1769) }\end{array}$ & SM & Recorded during 2011-12 \\
\hline 17 & Gadwall & $\begin{array}{l}\text { Anas strepera } \\
\text { Linnaeus, } 1758\end{array}$ & WM & Recorded during 2005-2008 \\
\hline 18 & Eurasian Wigeon & $\begin{array}{l}\text { Anas penelope } \\
\text { Linnaeus, } 1758\end{array}$ & WM & Recorded during 2005-2008 \\
\hline 19 & Mallard & $\begin{array}{l}\text { Anas platyrhynchos } \\
\text { Linnaeus, } 1758\end{array}$ & WM & Recorded during 2005-2008 \\
\hline 20 & Spot-billed Duck & $\begin{array}{l}\text { Anas poecilorhyncha } \\
\text { J.R. Forester, } 1781\end{array}$ & WM & $\begin{array}{l}\text { Recorded throughout the study } \\
\text { period }(2005-2012)\end{array}$ \\
\hline 21 & Northern Shoveller & $\begin{array}{l}\text { Anas clypeata } \\
\text { Linnaeus, } 1758\end{array}$ & WM & $\begin{array}{l}\text { Recorded throughout the study } \\
\text { period }(2005-2012)\end{array}$ \\
\hline 22 & Northern Pintail & $\begin{array}{l}\text { Anas acuta } \\
\text { Linnaeus, } 1758\end{array}$ & WM & $\begin{array}{l}\text { Recorded throughout the study } \\
\text { period (2005-2012) }\end{array}$ \\
\hline 23 & Garganey & $\begin{array}{l}\text { Anas querquedula } \\
\text { Linnaeus, } 1758\end{array}$ & WM & Recorded during 2005-2008 \\
\hline
\end{tabular}


Table 1. Contd.

\begin{tabular}{|c|c|c|c|c|}
\hline 24 & Common Teal & $\begin{array}{l}\text { Anas crecca } \\
\text { Linnaeus, } 1758\end{array}$ & WM & $\begin{array}{l}\text { Recorded throughout the study } \\
\text { period (2005-2012) }\end{array}$ \\
\hline 25 & Common Pochard & $\begin{array}{l}\text { Aythya ferina } \\
\text { (Linnaeus, 1758) }\end{array}$ & WM & Recorded during 2005-2008 \\
\hline & Gruiformes & \multicolumn{3}{|c|}{ Rallidae } \\
\hline 26 & $\begin{array}{l}\text { White-breasted } \\
\text { Waterhen }\end{array}$ & $\begin{array}{l}\text { Amaurornis phoenicurus } \\
\text { (Pennant, 1769) }\end{array}$ & $\mathrm{R}$ & $\begin{array}{l}\text { Recorded throughout the study } \\
\text { period }(2005-2012)\end{array}$ \\
\hline 27 & Purple Moorhen & $\begin{array}{l}\text { Porphyrio porphyrio } \\
\text { (Linnaeus, 1758) }\end{array}$ & $\mathrm{R}$ & $\begin{array}{l}\text { Recorded throughout the study } \\
\text { period (2005-2012) }\end{array}$ \\
\hline 28 & Common Moorhen & $\begin{array}{l}\text { Gallinula chloropus } \\
\text { (Linnaeus, 1758) }\end{array}$ & LM & $\begin{array}{l}\text { Recorded throughout the study } \\
\text { period (2005-2012) }\end{array}$ \\
\hline \multirow[t]{2}{*}{29} & Common Coot & $\begin{array}{l}\text { Fulica atra } \\
\text { Linnaeus, } 1758\end{array}$ & WM & $\begin{array}{l}\text { Recorded throughout the study } \\
\text { period (2005-2012) }\end{array}$ \\
\hline & Charadriiformes & & \multicolumn{2}{|c|}{ Jacanidae } \\
\hline 30 & Pheasant-tailed Jacana & $\begin{array}{l}\text { Hydrophasianus chirurgus } \\
\text { (Scopoli, 1786) }\end{array}$ & SM & Recorded during 2009-2012 \\
\hline \multirow[t]{2}{*}{31} & Bronze-winged Jacana & $\begin{array}{l}\text { Metopidius indicus } \\
\text { (Latham, 1790) }\end{array}$ & $\mathrm{R}$ & Recorded during 2009-2012 \\
\hline & & \multicolumn{3}{|c|}{ Charadriidae } \\
\hline 32 & Little Ringed Plover & $\begin{array}{l}\text { Charadrius dubius } \\
\text { Scopoli, } 1786\end{array}$ & WM & Recorded during 2005-2008 \\
\hline 33 & Kentish Plover & $\begin{array}{l}\text { Charadrius alexandrinus } \\
\text { Linnaeus, } 1758\end{array}$ & WM & Recorded during 2005-2008 \\
\hline 34 & $\begin{array}{l}\text { Yellow-wattled } \\
\text { Lapwing }\end{array}$ & $\begin{array}{l}\text { Vanellus malabaricus } \\
\text { (Boddaert, 1783) }\end{array}$ & $\mathrm{R}$ & Recorded during 2005-2008 \\
\hline 35 & Red-wattled Lapwing & $\begin{array}{l}\text { Vanellus indicus } \\
\text { (Boddaert, 1783) }\end{array}$ & $\mathrm{R}$ & $\begin{array}{l}\text { Recorded throughout the study } \\
\text { period (2005-2012) }\end{array}$ \\
\hline \multirow[t]{2}{*}{36} & White-tailed Lapwing & $\begin{array}{l}\text { Vanellus leucurus } \\
\text { (Lichtenstein, 1823) }\end{array}$ & WM & Recorded during 2009-2012 \\
\hline & & \multicolumn{3}{|c|}{ Scolopacidae } \\
\hline 37 & Spotted Redshank & $\begin{array}{l}\text { Tringa erythropus } \\
\text { (Pallas, 1764) }\end{array}$ & WM & Recorded during 2005-2008 \\
\hline 38 & Common Redshank & $\begin{array}{l}\text { Tringa totanus } \\
\text { (Linnaeus, 1758) }\end{array}$ & WM & $\begin{array}{l}\text { Recorded throughout the study } \\
\text { period }(2005-2012)\end{array}$ \\
\hline 39 & Green Sandpiper & $\begin{array}{l}\text { Tringa ochropus } \\
\text { Linnaeus, } 1758\end{array}$ & WM & Recorded during 2005-2008 \\
\hline 40 & Common Sandpiper & $\begin{array}{l}\text { Actitis hypoleucos } \\
\text { Linnaeus, } 1758\end{array}$ & WM & $\begin{array}{l}\text { Recorded throughout the study } \\
\text { period (2005-2012) }\end{array}$ \\
\hline 41 & Little Stint & $\begin{array}{l}\text { Calidris minuta } \\
\text { (Leisler, 1812) }\end{array}$ & WM & Recorded during 2005-2008 \\
\hline 42 & Ruff & $\begin{array}{l}\text { Philomachus pugnax } \\
\text { (Linnaeus, 1758) }\end{array}$ & WM & Recorded during 2005-2008 \\
\hline 43 & Marsh Sandpiper & $\begin{array}{l}\text { Tringa stagnatilis } \\
\text { (Bechstein, 1803) }\end{array}$ & WM & $\begin{array}{l}\text { Recorded throughout the study } \\
\text { period }(2005-2012)\end{array}$ \\
\hline 44 & Common Greenshank & $\begin{array}{l}\text { Tringa nebularia } \\
\text { (Gunner, 1767) }\end{array}$ & WM & $\begin{array}{l}\text { Recorded throughout the study } \\
\text { period (2005-2012) }\end{array}$ \\
\hline 45 & Wood Sandpiper & $\begin{array}{l}\text { Tringa glareola } \\
\text { (Linnaeus, 1758) }\end{array}$ & WM & $\begin{array}{l}\text { Recorded throughout the study } \\
\text { period (2005-2012) }\end{array}$ \\
\hline \multirow[t]{2}{*}{46} & Pintail Snipe & $\begin{array}{l}\text { Gallinago stenura } \\
\text { (Bonaparte, 1830) }\end{array}$ & WM & Recorded during 2009-2012 \\
\hline & & \multicolumn{3}{|c|}{ Recurvirostridae } \\
\hline 47 & Black-winged Stilt & $\begin{array}{l}\text { Himantopus himantopus } \\
\text { (Linnaeus, 1758) }\end{array}$ & $\mathrm{R}$ & $\begin{array}{l}\text { Recorded throughout the study } \\
\text { period (2005-2012) }\end{array}$ \\
\hline
\end{tabular}


Table 1. Contd.

\begin{tabular}{|c|c|c|c|c|}
\hline 48 & Pied Avocet & $\begin{array}{l}\text { Recurivirostra avosetta } \\
\text { Linnaeus, } 1758\end{array}$ & WM & Recorded during 2005-2008 \\
\hline & & \multicolumn{3}{|c|}{ Laridae } \\
\hline \multirow[t]{2}{*}{49} & River Tern & $\begin{array}{l}\text { Sterna aurantia } \\
\text { J.E.Gray, } 1831\end{array}$ & LM & Recorded during 2005-2008 \\
\hline & Falconiformes & \multicolumn{3}{|c|}{ Accipitridae } \\
\hline \multirow[t]{2}{*}{50} & Brahminy Kite & $\begin{array}{l}\text { Haliastur indus } \\
\text { (Boddaert, 1783) }\end{array}$ & $\mathrm{R}$ & $\begin{array}{l}\text { Recorded throughout the study } \\
\text { period (2005-2012) }\end{array}$ \\
\hline & Coraciiformes & \multicolumn{3}{|c|}{ Alcedinidae } \\
\hline 51 & Lesser Pied Kingfisher & $\begin{array}{l}\text { Ceryle rudis } \\
\text { (Linnaeus, 1758) }\end{array}$ & $\mathrm{R}$ & $\begin{array}{l}\text { Recorded throughout the study } \\
\text { period }(2005-2012)\end{array}$ \\
\hline 52 & $\begin{array}{l}\text { White-breasted } \\
\text { Kingfisher }\end{array}$ & $\begin{array}{l}\text { Halcyon smyrnensis } \\
\text { (Linnaeus, 1758) }\end{array}$ & $\mathrm{R}$ & $\begin{array}{l}\text { Recorded throughout the study } \\
\text { period }(2005-2012)\end{array}$ \\
\hline & Apodiformes & \multicolumn{3}{|c|}{ Apodidae } \\
\hline 53 & House Swift & $\begin{array}{l}\text { Apus affinis } \\
\text { (J.E.Gray,1830) }\end{array}$ & $\mathrm{R}$ & $\begin{array}{l}\text { Recorded throughout the study } \\
\text { period (2005-2012) }\end{array}$ \\
\hline & Passeriformes & \multicolumn{3}{|c|}{ Hirundinidae } \\
\hline 54 & Wire-tailed Swallow & $\begin{array}{l}\text { Hirundo smithii } \\
\text { Leach, } 1818\end{array}$ & $\mathrm{R}$ & $\begin{array}{l}\text { Recorded throughout the study } \\
\text { period }(2005-2012)\end{array}$ \\
\hline 55 & Common Swallow & $\begin{array}{l}\text { Hirundo rustica } \\
\text { Linnaeus, } 1758\end{array}$ & $\mathrm{R}$ & $\begin{array}{l}\text { Recorded throughout the study } \\
\text { period (2005-2012) }\end{array}$ \\
\hline & & \multicolumn{3}{|c|}{ Motacillidae } \\
\hline 56 & White Wagtail & $\begin{array}{l}\text { Motacilla alba } \\
\text { Linnaeus, } 1758\end{array}$ & WM & $\begin{array}{l}\text { Recorded throughout the study } \\
\text { period (2005-2012) }\end{array}$ \\
\hline 57 & Large Pied Wagtail & $\begin{array}{l}\text { Motacilla maderaspat- } \\
\text { ensis } \\
\text { Gmelin, } 1789\end{array}$ & LM & $\begin{array}{l}\text { Recorded throughout the study } \\
\text { period }(2005-2012)\end{array}$ \\
\hline 58 & Citrine Wagtail & $\begin{array}{l}\text { Motacilla citreola } \\
\text { (Pallas, 1776) }\end{array}$ & WM & $\begin{array}{l}\text { Recorded throughout the study } \\
\text { period }(2005-2012)\end{array}$ \\
\hline 59 & Yellow Wagtail & $\begin{array}{l}\text { Motacilla flava } \\
\text { (Linnaeus, 1758) }\end{array}$ & WM & $\begin{array}{l}\text { Recorded throughout the study } \\
\text { period }(2005-2012)\end{array}$ \\
\hline
\end{tabular}

R-Resident; WM-Winter migratory; LM-Local migratory and SM-Summer migratory.

Anas strepera, Eurasian Wigeon Anas penelope, White -tailed Lapwing Vanellus leucurus, Green Sandpiper Tringa ochropus, Common Greenshank Tringa nebularia, Openbill Stork Anastomus oscitans etc. Local migratory birds like Little Cormorants Phalacrocorax niger, Median Cormorants Phalacrocorax fuscicollis and Large Cormorants Phalacrocorax carbo were in impressive number until 2008AD (table-1). The scenario observed in 2009-10 winter season was that no migratory birds were seen (Table 1). Pond Herons decreased in number by a margin of $90 \%$. The same situation was seen in terms of Grey Herons, Common Coot, Cattle Egrets, Intermediate Egrets, Purple Heron, Northern Shoveller, Northern Pintail, Common Teal, Common Pochard, Garganey and Gadwall. The number of Pond Herons has decreased. Contrary to this, the number of Bronze Winged Jacanas has increased tremendously, indicating the luxurious growth of hyacinth and giving direct hint of last stage of eutrophication (Figs1-3, Plate-1).

It is astonishing to observe increasing number of Common Teal, Northern Shoveller, White-tailed Lapwing, Brahminy Shelduck, Pintail Snipe Gallinago stenura, Common Coot, and Northern Pintail which are wetland birds of Siberia, Russia, China, Tibet and other trans-Himalayan regions during winter season of 2012-13. The highest number in December, 2012 refers to those of Common Teal, followed by Northern Shoveller, Northern Pintail and white-tailed Lapwing. In addition, visit in early February, 2013 confirms the presence of Rudy Shelduck, Common Teal, Garganey, Common Redshank, Common Sandpiper, Northern Pintail, Gadwall and Spot-billed Duck. The Mallard, the flagship species of migratory birds were totally absent in the winter of 2011-12 (Table 1).

It is to be noted over here that this Palwal-pond was a ready means to receive rainy water and recharge the underground water table of the village. It was a 


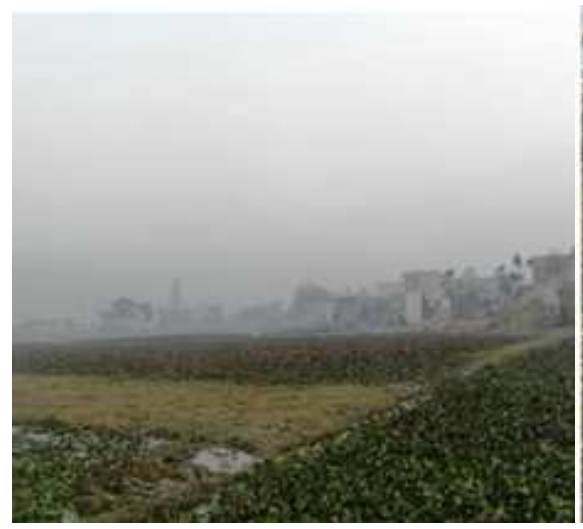

Fig. 1. Palwal village pond

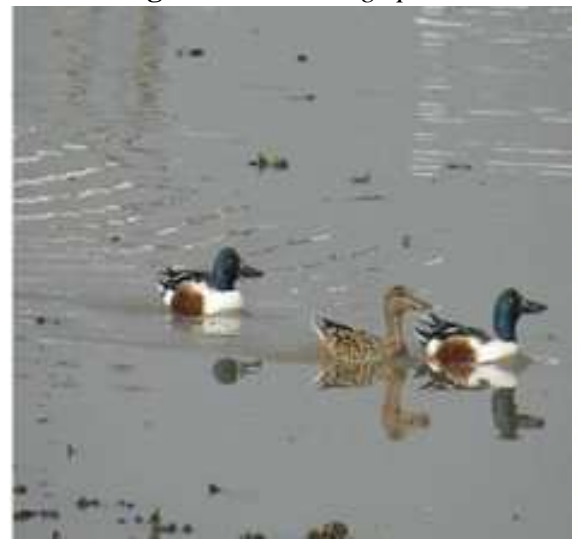

Fig. 4. Northern Shovellers

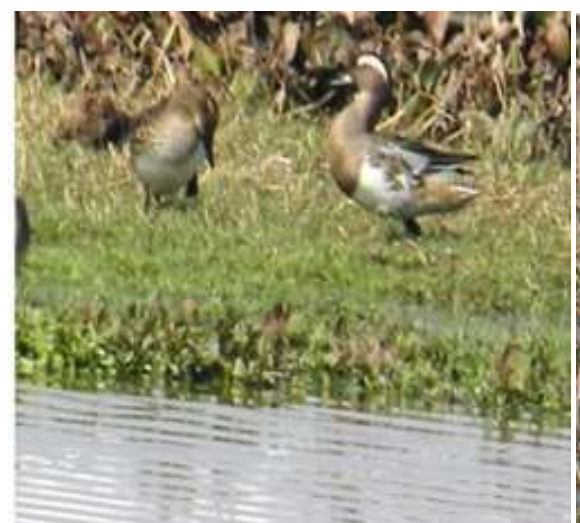

Fig. 7. A pair of Garganey

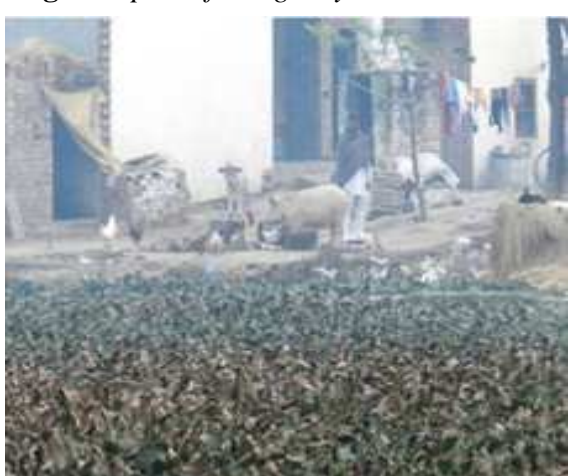

Fig. 10. Encroachment of pond by the society

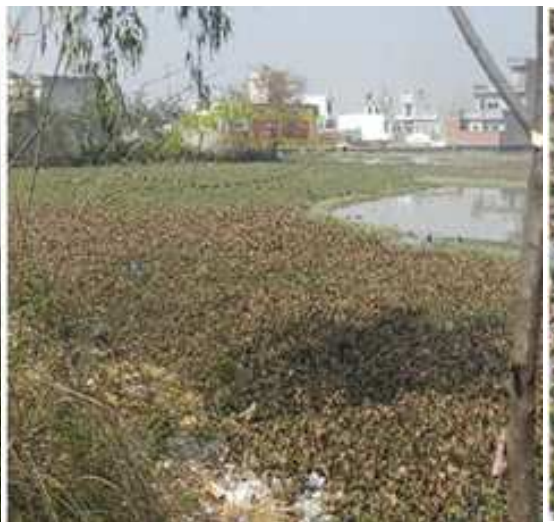

Fig. 2. Hyacinth proliferation

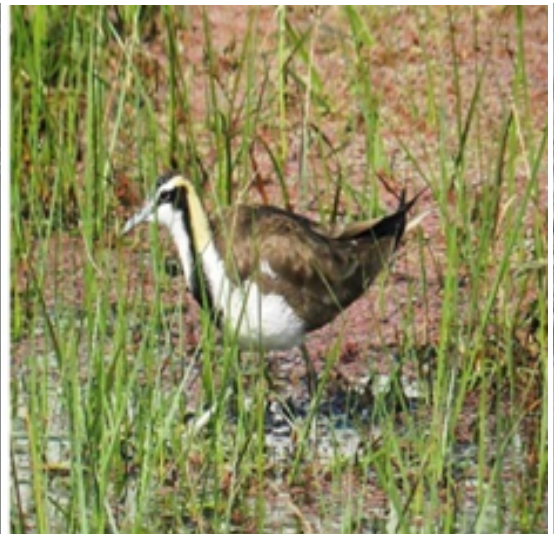

Fig. 5. Pheasant - tailed Jacana

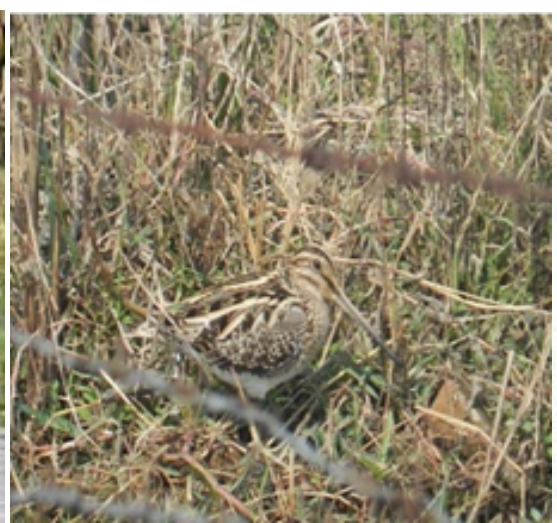

Fig. 8. Pintail snipe

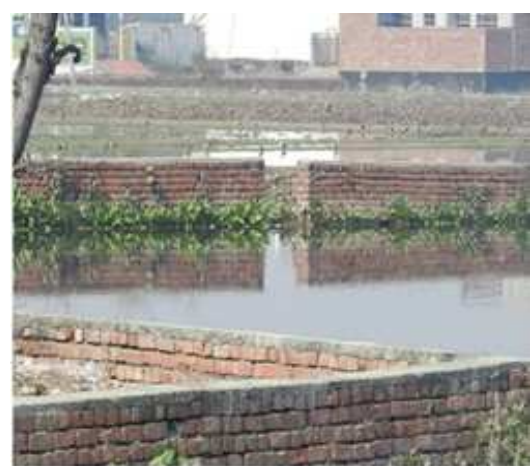

Fig. 11. Encroachment of pond by the society

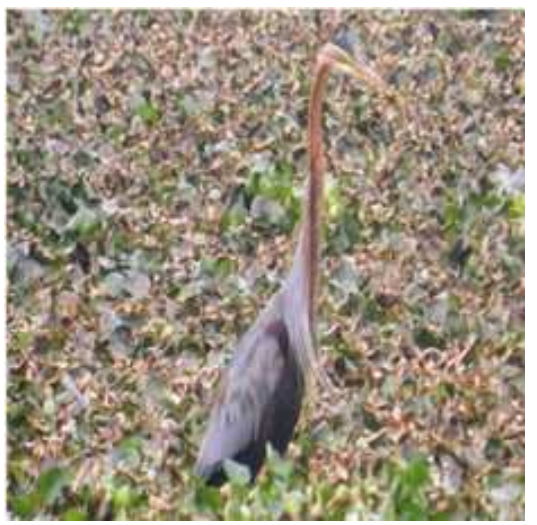

Fig. 3. Purple heron

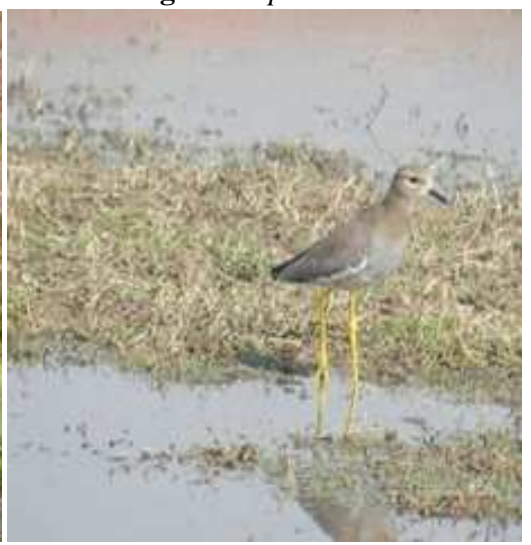

Fig. 6. White tailed lapwing

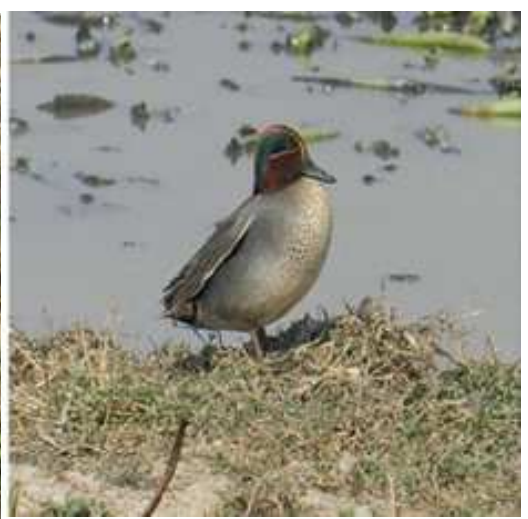

Fig. 9. Common Teal

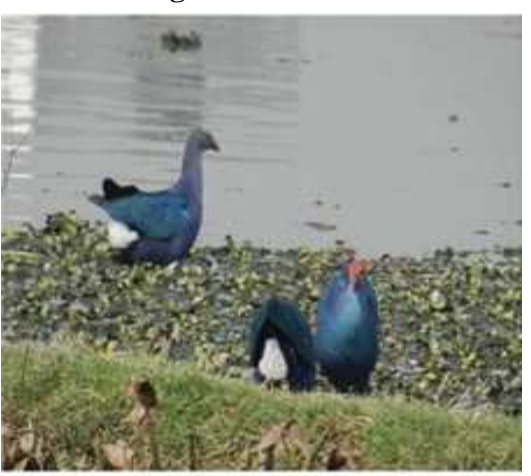

Fig. 12. Purple Moorhens

Plate 1. Photographic inventory of scenario prevalent at Palwal village pond in Kurukshetra district in Haryana during study period. 
multipurpose village pond for villagers, farmers and "Prajapatis". Villagers will draw drinking water from nearby villages which are totally dry today. Farmers would draw irrigation water. Prajapatis will get soft-soil (Chikni-Mitti) to make mud pitchers and bricks for Kilns. Poor villagers will get free Kachhi-Matti to reinforce "roofs" of kachha houses before the onset of rainy season. The domestic cattle would find a heavenly resort in this pond for bathing; drinking and necessary out-door activity filled refreshing bouts. It was an exampling nicely weaven network of a sustainable activity linking man, water, rains, birds, fish, livestock and rural cottage industry of KILNS.

Today, the entire scene stands shattered. A vast sheet of water; a huge natural resource born out of rainy water, a free of cost resort for precious villagers' livestock, a ready means of revitalizing the underground water-sadly faces its demise. It is, certainly, not an isolated case. The Palwal-Pond episode of degradation of the precious water resource can be safely extended to each and every village of Haryana, Rajasthan, Uttar Pradesh, Bihar, West-Bengal, Jharkhand, and Chhattisgarh. The situation is adverse. Wetlands of villages are depleting at a fast rate. Given the present situation, there would no more traditional village Kunds, Talabs, Khappars, Pokhars, Sarovars in rural India.

\section{Conclusion}

The migratory wetland birds in rural ponds are facing extreme conditions due to destruction of age old rural ponds in Haryana. It is serious issue which has very easy solution lying at the next door. A well known scheme for rural upliftment programme run by Govt. of India, namely, Mahatma Gandhi National Rural Employment Guarantee Act can prevent the depletion of village ponds, probably reversed within just one year. Our village ponds will restart serving as the wintering grounds for migratory birds coming to Haryana from Siberia, Tibet, China, Ladhakh, Central Asia etc.

\section{ACKNOWLEDGEMENTS}

The authors are thankful to authorities of Kurukshetra University including Chairman, Department of Zoology, Kurukshetra University, Kurukshetra for cooperation, encouragement and inspiration

\section{REFERENCES}

Ali, S. (1996). The Book of Indian Birds. $12^{\text {th }}$ Edition (Revised \& enlarged): Oxford University Press, Mumbai.

Ali, S. and Ripley, S. D. (1987). Handbook of the birds of India and Pakistan together with those of Bangladesh, Nepal, Bhutan and Sri Lanka.1- 10 Vols. Oxford University Press. New Delhi.

Grimmet, R., Inskipp, T. and Inskipp, C. (1998). Birds of the Indian subcontinent. Oxford University Press, Delhi: pp. 888

Gupta, R.C. and Kaushik, T.K. (2010a). Determination of the domain of spectrum concerning diversity of endangered winter visitor wetland birds in Haryana. Journal of Experimental Zoology, India, 13 (2):349354.

Gupta, R.C. and Kaushik, T.K. (2010b). Computation of wetland birds in rural areas of Kurukshetra, Haryana, India. Journal of Nature Conservation, 22 (1):1-11.

Gupta, R.C. and Kaushik, T.K. (2010c). On the causative factors responsible for the pathetic plight of Yellow wattled Lapwing in Kurukshetra suburbs. Journal of Nature Conservation, 22 (2):181-187.

Gupta, R.C. and Kaushik, T.K. (2010d). Determination of spectrum of winter migratory birds in Yamunanagar district in Haryana (India). Environment Conservation Journal, 11(3):37-43.

Gupta, R.C. and Kaushik, T.K. (2010e). Understanding Rural Ponds' Migratory Avian Diversity in Panchkula District in Haryana, India. Journal of Advanced Zoology, 31 (2):117-123.

Gupta, R.C. and Kaushik, T.K. (2011a). On the fast depleting trends of Cormorants in Kurukshetra wetlands in the last twenty five years. Journal of Experimental Zoology, India, 14 (1):81-85.

Gupta, R.C. and Kaushik, T.K. (2011b). On the fundamentals of natural history and present threats to Red-wattled Lapwing in Kurukshetra environs. Journal of Applied and Natural Science, 3(1):62-67.

Gupta, R.C. and Kaushik, T.K. (2012). A case study: to demonstrate total destruction of a vibrant rural pond: hub for over hundred winter migratory birds up to $2005 \mathrm{AD}$. Life Science Leaflets, 4: 1-11.

Gupta, R.C. and Kaushik, T.K. (2013a). Discussing implications of fast depleting rural ponds on the globally threatened wetland winter migratory bird in Haryana: A case study of Nigdu village pond in district Karnal. Journal of Tropical Life Sciences, 3 (2):1-9.

Gupta, R.C. and Kaushik, T.K. (2013b). Rich winter migratory birds in thousands have become victim of Kurukshetra Utsav in Haryana, India. International Journal of life sciences 7(1):6-11.

Gupta, R.C., Kaushik, T.K. and Kumar, S. (2010a). Evaluation of the extent of wetland birds in district Kaithal, Haryana, India. Journal of Applied and Natural Science, 2(1):77-84.

Gupta, R.C., Kaushik, T.K. and Kumar, S. (2010b). An account concerning arrival and departure time of few selected winter migratory birds in Haryana rural ponds. Environment Conservation Journal, 11(1\&2): 1-9.

Gupta, R.C., Kaushik, T.K. and Gupta, P.K. (2012). Winter migratory wetland birds in Haryana are confronting adverse conditions in rural ponds resulting in reduction in arrival number: A case study of village Amin in Thanesar block in Kurukshetra district. Indian Journal of Fundamental and Applied Life Sciences, 2 (1): 1-7.

Gupta, R.C., Kaushik, T.K. and Kumar, S. (2009). Analysis of winter migratory wetland birds in Karnal district in Haryana. Journal of Advanced Zoology, 30 (2):104-117.

Gupta, R.C., Kumar, S. and Kaushik, T.K. (2010c). Computation of route specific Avi-faunal diversity in Morni Hills in Panchkula district in Haryana State in India. Journal of Advanced Zoology, 31 (1):1-9.

Kaushik, T.K. and Gupta, R.C. (2013). Understanding and analysing the coordinates of diversity of wetland birds of Asan barrage near Paonta Sahib, Northern India. Our 
Nature, 11(2): 192-200.

Kaushik, T.K. and Gupta, R.C. (2014). Black Kite populations are suffering declining trends in Kurukshetra and likely to get further depleted-an analysis of causes. Journal of Tropical Life Sciences, 4 (1):14-18.

Kumar, A., Sati, J.P., Tak, P.C. and Alfred, J.R.B. (2005).
Handbook on Indian Wetland Birds and their Conservation: i-xxvi; 1-468: Published by Director, Zool. Surv. India.

Manakadan, R. and Pittie, A. (2001). Standardized common and scientific names of the birds of Indian subcontinent. Buceros, 6(1): i-ix, 1-38 